\title{
Alfvén waves in space and astrophysical dusty plasmas
}

\author{
V. Jatenco-Pereira ${ }^{1}$, A. C.-L. Chian ${ }^{2,3,4}$, and N. Rubab ${ }^{5}$ \\ ${ }^{1}$ Instituto de Astronomia, Geofísica e Ciências Atmosféricas (IAG/USP), Rua do Matão, 1226, 05508-090 São Paulo, SP, \\ Brazil \\ ${ }^{2}$ Paris Observatory, LESIA, CNRS, 92190 Meudon, France \\ ${ }^{3}$ National Institute for Space Research (INPE) and World Institute for Space Environment Research (WISER), P.O. Box 515, \\ 12227-010 São José dos Campos, SP, Brazil \\ ${ }^{4}$ School of Mathematical Sciences, University of Adelaide, Adelaide, SA 5005, Australia \\ ${ }^{5}$ Department of Space Science, Institute of Space Technology (IST), Islamabad, Pakistan \\ Correspondence to: V. Jatenco-Pereira (vera.jatenco@iag.usp.br)
}

Received: 9 August 2013 - Revised: 21 November 2013 - Accepted: 28 January 2014 - Published: 13 March 2014

\begin{abstract}
In this paper, we present some results of previous works on Alfvén waves in a dusty plasma in different astrophysical and space regions by taking into account the effect of superthermal particles on the dispersive characteristics. We show that the presence of dust and superthermal particles sensibly modify the dispersion of Alfvén waves. The competition between different damping processes of kinetic Alfvén waves and Alfvén cyclotron waves is analyzed. The nonlinear evolution of Alfvén waves to chaos is reviewed. Finally, we discuss some applications of Alfvén waves in the auroral region of space plasmas, as well as stellar winds and star-forming regions of astrophysical plasmas.
\end{abstract}

\section{Introduction}

The dusty plasma has been actively investigated in the past few decades and a number of its applications have been found in laboratory systems (e.g., cold atmospheric plasma for cancer therapy, Keidar et al., 2013; Köritzer et al., 2013; Walk et al., 2013) as well as in space and astrophysical systems (e.g., auroral regions, stellar winds and star-forming regions). The aim of this paper is to review some recent advances in the study of parallel propagation of Alfvén waves and kinetic Alfvén waves in space and astrophysical dusty plasmas as well as nonlinear evolution of Alfvén waves to chaos. The layout of the manuscript is organized as follows: an overview of the theory of Alfvén waves and dispersion characteristics in dusty plasmas is presented in Sect. 2.1. In Sect. 2.2, we discussed the damping of kinetic Alfvén waves. Section 2.3 is devoted to a short overview of the Lorentzian particle velocity distribution function and its effects on Alfvén waves. The nonlinear evolution of large-amplitude Alfvén waves to chaos is analyzed in Sect. 2.4. Various space and astrophysical applications of the interactions of Alfvén waves with dust plasma particles are discussed in Sects. 3 and 4, respectively. Finally, a brief summary is given in Sect. 5 .

\section{Alfvén waves in dusty plasmas}

Dusty plasmas are ionized gases in the presence of microparticles and often are termed as "complex plasmas" or "colloidal plasmas". These dust particles, or dust grains, are highly massive and usually negatively charged due to the higher mobility and the large flux of incoming electrons, and therefore an isolated dust grain in a plasma acts like a floating probe. Orbital motion limited (OML) is one of the ways to determine the charging cross-section on a grain surface pertaining to the laws of conservation of energy and momentum. For an isolated dust grain it can be formulated as $a \ll$ $\lambda_{D} \ll d$ where $a$ is the dust grain size, $d$ is the the grain average separation $\left(d=\left(3 / 4 \pi n_{d 0}\right)^{1 / 3}\right.$, where $n_{d 0}$ is the grain number density) and the dusty plasma Debye length of $\beta$ species particles $\left(\lambda_{D \beta 0}=\left(k_{B} T_{\beta} / 4 \pi n_{\beta 0} q_{\beta}^{2}\right)^{1 / 2}\right.$, where $k_{B}$ is the Boltzmann constant). In the case under consideration, we always have $a \ll \lambda_{D \beta 0}$ and for dusty plasma $d \leq \lambda_{D \beta 0}$ and the dust particles can be treated as massive point particles where the effect of neighboring particles is expressive and we can expect collective behavior (de Angelis, 1992). Dusty plasmas are present in many astrophysical environments, as 
for example the rings of Saturn, cometary tails or interstellar clouds (Goertz, 1989; Verheest, 2000). The grain size is not uniform in many space and astrophysical dusty plasmas and can vary from sub-micron to centimeter scale; therefore, one can consider different dust size distributions. It is well known that the presence of dust particles significantly modifies the electrostatic/electromagnetic modes due to quasi-neutrality and is responsible for some new modes that are associated with dusty dynamics, and the coupling of these tiny dust grains to the magnetic field is very important to determine the wave propagation in the planetary and interstellar media, i.e., ions and dust micro-particulates provide an effective coupling between Alfvén wave and neutral gas in the molecular clouds (Elmegreen and Fiebig, 1993).

\subsection{Dispersion relation of Alfvén waves in a dusty plasma}

It is well known that the properties of Alfvén and magnetoacoustic waves are radically modified in a dusty medium, especially when the charge on the dust grains fluctuates; when the charged grains acquire a circular motion around the field lines that is defined by the dust cyclotron frequency; and when the waves and the grains experience the same frequency, a resonance occurs that leads to the damping of the waves.

Various modes of propagation in a dusty plasma arise from the fact that frequencies associated with dust particles are smaller than those for electrons and ions. These waves are ultra-low frequency dust modes (Rao, 1996) and are associated with the dust particle inertia. Here, charged dust grains have a collective behavior and take part in the wave dynamics. As examples of these oscillation modes, we could mention dust acoustic waves (DAW) (Rao et al., 1990; D' Angelo, 1995) and electrostatic dust-cyclotron waves (EDCW) (Sorasio and Rosenberg, 2001). Magnetized dusty plasmas support additional electrostatic low-frequency waves involving the dynamics of magnetized/unmagnetized dust grains and magnetized electrons/ions, i.e., dust lower hybrid (DLH) waves with $\omega \ll \omega_{\mathrm{ci}}$, which propagate nearly perpendicular to the external magnetic field.

The dispersion relation of Alfvén waves, with frequencies smaller than the ion cyclotron frequency, assuming constant charged particles in a neutral and cold dusty plasma, is given by (Cramer et al., 2002)

$k_{z}^{2}=u_{1} \pm u_{2}$,

where

$u_{1}=\frac{\omega^{2} \Omega_{\mathrm{i}}^{2}}{v_{\mathrm{Ai}}^{2}\left(\Omega_{\mathrm{i}}^{2}-\omega^{2}\right)}+\frac{\omega^{2} \omega_{\max }^{2}}{s v_{\mathrm{Ad}}^{2}} \int_{1}^{a_{\mathrm{m}}} \frac{f(\mathcal{R}) \mathrm{d} \mathcal{R}}{\mathcal{R}\left(\omega_{\max }^{2} / \mathcal{R}^{4}-\omega^{2}\right)}$, and

$u_{2}=\frac{\omega^{3} \Omega_{\mathrm{i}}}{v_{\mathrm{Ai}}^{2}\left(\Omega_{\mathrm{i}}^{2}-\omega^{2}\right)}+\frac{\omega^{3} \omega_{\max }}{s v_{\mathrm{Ad}}^{2}} \int_{1}^{a_{\mathrm{m}}} \frac{\mathcal{R} f(\mathcal{R}) \mathrm{d} \mathcal{R}}{\omega_{\max }^{2} / \mathcal{R}^{4}-\omega^{2}}$.

In the equations $\omega$ is the angular wave frequency, $\Omega_{\mathrm{i}}$ is the ion cyclotron frequency, $v_{\mathrm{Ai}}$ and $v_{\mathrm{Ad}}$ are the Alfvén speeds of the ions and dust, respectively, $s=c_{4} \ln \left(a_{\mathrm{m}}\right)$ where $a_{\mathrm{m}}=a_{\max } / a_{\min }\left(a_{\min }\right.$ and $a_{\max }$ are the minimum and the maximum dust radii, respectively), $c_{4}=3 /\left(1-a_{\mathrm{m}}^{-3}\right)$ and $c$ is the speed of light. Also, $\omega_{\max }=q_{\min } B /\left(m_{\min } c\right)$ is the maximum dust cyclotron frequency (i.e., for the smallest dust grain with mass $m_{\min }$ and charge $\left.q_{\min }\right)$. The need for $m_{\min }$ and $q_{\min }$ comes from the fact that considering a Maxwellian velocity distribution for electrons and ions, in the equilibrium situation where the dust charge does not vary with time, the electric currents on the dust surface must be identical (Vladimirov, 1997). As a result, for grain radius $a>$ $10^{-7} \mathrm{~cm}$, the dust charge increases linearly with the grain size.

The right-hand polarized wave (positive sign in Eq. 1) is the mode damped by the dust resonance. In the case of $\omega_{\min }<\omega<\omega_{\max }$, the integral in the particle radii has singularities, whose residues give the complex part of the wave number $\left(k_{z} \equiv k_{\mathcal{R}}+i k_{\mathcal{C}}\right)$ and lead to the dust-cyclotron damping of the waves, with damping length $L \equiv L(\omega)=$ $2 \pi / k_{\mathcal{C}}(\omega)$. If we consider a distribution of grain sizes, the waves are damped in a broad band of resonance (Vidotto and Jatenco-Pereira, 2006).

\subsection{Damping of kinetic Alfvén waves}

Alfvén waves are a fundamental physical phenomenon in magnetized plasma that contributes to a wide variety of physical processes in space plasmas, for example, turbulence, plasma heating, acceleration along the field lines, wave particle interactions and generation of geomagnetic perturbations. The interaction of the wave with thermal ions is one of the important features in many astrophysical regions, such as solar corona/solar wind, for transporting thermal energy (Yukhimuk et al., 2000). Kinetic Alfvén waves (KAWs) are small-scale dispersive Alfvén waves, differ drastically from MHD Alfvén waves and appear when the cross field scales are comparable to ion gyroradius.

A kinetic Alfvén wave is an important electromagnetic wave that transports electromagnetic energy in many space and astrophysical regions (Nakano et al., 2002; Voitenko et al., 2003). The KAW can transfer the wave energy to electrons via Landau damping, which is the collisionless damping of low-frequency waves that occurs when thermal electrons travel along the magnetic field lines. In this process the particles gain kinetic energy at the expense of the wave if the distribution function has a negative slope in the range $v \sim$ $\omega / k$ and it results in the heating of plasmas or acceleration of electrons along the magnetic field direction (Cramer, 2001). 
In many space and astrophysical regions, the wave properties such as the wave dispersion and the onset of Landau/cyclotron damping are dependent on the plasma beta (Gary and Borowsky, 2004).

The wave-particle interaction in KAWs depends sensitively on the plasma $\beta$ parameter (Wu et al., 1996). Hasegawa and Cheng (1976) showed that in a low beta plasma $1>\beta>$ $m_{\mathrm{e}} / m_{\mathrm{i}}$, the wave is weakly damped due to ion Landau damping leading to ion heating (Hasegawa and Cheng, 1976), whereas if electron temperature is comparable to ion temperature, nonlinear Landau damping occurs. For some parameter regimes where $\beta \sim m_{\mathrm{e}} / m_{\mathrm{i}}$, the strong electron Landau damping will lead to electron heating (Wu and Fang, 1999).

The dissipation of KAWs by electron Landau damping has been suggested to play a significant role in the damping of interplanetary magnetic field fluctuations and to explain the slope of the magnetic turbulence spectrum where the spectral break is believed to cause the collisionless damping at short scales, which converts the turbulent energy into thermal energy.

Zhao et al. (2011) discussed the damping mechanism of KAWs in the dense plume structure of coronal holes and showed that KAWs are strongly damped in this region due to Landau damping when $\lambda_{\mathrm{e}} k_{\perp}<0.3$.

Wu and Fang $(1999,2003,2007)$ and Wu et al. (1996) showed that the parallel electric field of the KAW can lead to effective electron heating due to kinetic damping or Ohmic dissipation, which can further dissipate the KAW energy to sustain the temperature of the coronal loop plasmas. This dissipation occurs due to the parallel electric field of the KAW, which helps the local electron heating and acceleration mechanism to balance the additional radiative loss of the bright plume.

The presence of dust grains in many space/astrophysical regions may also play an important role in the dynamics of Alfvén waves, i.e., breaking down of frozen approximation in field conditions (Kamaya and Nishi, 2000). The coupling of charged dust grains to plasma through electric or magnetic fields can affect the spectrum of low-frequency waves through dust inertia and cyclotron resonance. The damping of KAWs in dusty plasma has been studied rigorously by many authors. Das et al. (1996) studied the KAW by retaining the finite Larmor radius of dust grains and showed that unlike MHD Alfvén waves, the KAW propagate across the magnetic field and because of its coupling to electrostatic modes, i.e., dust acoustic mode, and it undergoes Landau damping for each plasma species (Das et al., 1996)

$$
\begin{aligned}
& \omega^{2}=k_{\|}^{2} V_{\mathrm{DA}}^{2}\left[1+k_{\perp}^{2} \rho_{\mathrm{d}}^{2}\left(\frac{3}{4}+\frac{T_{\mathrm{e}}}{T_{\mathrm{d}}}\right)\right. \\
&\left. \pm \frac{i \pi n_{\mathrm{d} 0} \omega_{\mathrm{cd}}^{2}\left(\frac{r_{\mathrm{d}}^{2}}{\lambda_{\text {Dio }}^{2}}+\frac{r_{\mathrm{d}}^{2}}{\lambda_{\text {Deo }}^{2}}\right)\left(1+\frac{3}{4} k_{\perp}^{2} \rho_{\mathrm{d}}^{2}\right)}{\omega_{\mathrm{pd}}^{2} k_{\|}\left(\omega-k_{\|} v_{\|}\right)}\right],
\end{aligned}
$$

where $V_{\mathrm{DA}}=c \omega_{\mathrm{cd}} / \omega_{\mathrm{pd}}$ is the dust Alfvén speed, $r_{\mathrm{d}}$ is the radius of dust grains, $\rho_{\mathrm{d}}=C_{\mathrm{D}} / \omega_{\mathrm{cd}},\left(C_{D}=\sqrt{T_{\mathrm{e}} / m_{\mathrm{d}}}\right)$ is the dust Larmor radius and $\omega_{\mathrm{cd}}=Q_{\mathrm{d} 0} B_{0} / m_{\mathrm{d}}$ is the dust cyclotron frequency with $Q_{\mathrm{d} 0}$ being the mean charge of the dust particles.

Rubab et al. (2009) showed that in a dusty Lorentzian plasma the Alfvén wave is coupled to the dust acoustic wave and the damping due to dust charge fluctuation is insensitive to the form of distribution function in the ultra low-frequency regime

$$
\omega^{2}=k_{\|}^{2} V_{\mathrm{DA}}^{2}\left[1+\frac{2 \kappa-3}{2 \kappa-1}\left(1+\frac{\omega_{\mathrm{pd}}^{2}}{c^{2} k_{\perp}^{2}}\right) k_{\perp}^{2} \rho_{\mathrm{d}}^{2} \pm i \pi n_{\mathrm{d} 0} \frac{r_{\mathrm{d}}^{2}}{k_{\|}}\right],
$$

where $\rho_{\mathrm{d}}=C_{D} / \omega_{\mathrm{cd}},\left(C_{D}=\sqrt{T_{\mathrm{eff}} / m_{\mathrm{d}}}\right)$, and $T_{\text {eff }}=n_{\mathrm{d} 0} Z_{\mathrm{d}}^{2}\left(T_{\mathrm{e}} n_{\mathrm{i} 0}+T_{\mathrm{i}} n_{\mathrm{e} 0}\right) / n_{\mathrm{i} 0} n_{\mathrm{e} 0}$.

However, Gaelzer et al. (2010) focused on high-frequency regimes with superthermal character of electrons and reported a significant modification of the dispersive and absorption characteristics. The absorption coefficient increases due to kappa-distributed electrons, which in turn have a large effect on the Whistler mode.

Gaelzer et al. (2009) studied extensively the properties of oblique Alfvén waves in a Maxwellian plasma with dust charge fluctuation effects using kinetic theory. They focused on the competition between various damping mechanisms, like Landau damping and damping due to charge variations where inelastic collisional frequency between the dust and plasma species plays a vital role in the wave absorption and hence the damping rates.

Dust grains play a crucial role in most space/astrophysical regions and are found to affect the growth/damping of many electromagnetic waves drastically. In a collisional dusty plasma the damping rate is found to be proportional to the collision frequency between dust and ions (Yang et al., 2012). It has been shown by many authors (Khan et al., 2013; Zubia et al., 2007) that the collisionless Landau damping is negligibly small for nearly perpendicular propagation (small $k_{\|}$), while dust charge fluctuation damping is the main mechanism for the damping of kinetic Alfvén waves, which could be a reason for plasma heating. de Juli et al. (2005) also explained that the Landau damping is negligible for the transverse ordinary Alfvén waves propagating parallel to the magnetic field.

\subsection{Alfvén waves in Lorentzian plasmas}

Velocity distribution functions with suprathermal tails in space and astrophysical plasmas are ubiquitous and persistent features and have been confirmed by many spacecraft missions. In kinetic and thermodynamical equilibrium the Maxwell-Boltzmann distributions come out as a solution of the Boltzmann collision term. Nevertheless, in a collisionless system exhibiting strong correlation and interactions on large scales, the Fokker-Planck approach happens to be 
invalidated. Treumann (1999), in persuit of the same goal, established the kinetic foundation of Lorentzian statistical mechanics to show that power-law velocity space distributions are a particular thermodynamic equilibrium state. According to the Lorentzian statistical mechanics, the power or the spectral index contains the information about the tangible turbulent system whose entropy is believed to increase slowly due to internal dissipation processes to maintain a stationary state. When the correlations in the system are absent, the nonthermal distribution approaches the classical Boltzmann distribution.

In most of the theoretical investigations reported so far, the particle distribution has been assumed to be Maxwellian. Spacecraft measurements of electron energy spectra and in situ observations in the near-Earth space plasma clearly indicate the presence of suprathermal particle populations. These particles may arise due to the effect of external forces acting on the natural space environments or due to wave-particle interaction. The measurements of velocity space and energy distribution functions in most of the realistic situations differ significantly from Fokker-Planck solutions. These observed distributions contain a plentiful supply of suprathermal particles, i.e., particles that move faster than the thermal speed and are represented by the family of kappa or Lorentzian distribution, which obeys a power law in particle speed with high-energy tails. Such a distribution gives a best fit to the observed Maxwellian-like distributions. The presence of suprathermal particles has prompted a number of investigations (Treumann, 1999; Collier, 1993; Vasyliunas, 1968). The mathematical form of isotropic kappa distribution in the literature is

$$
f^{\kappa}(r, v)=\frac{n}{2 \pi\left(\kappa v_{\mathrm{th}}^{2}\right)} \frac{\Gamma(\kappa+1)}{\Gamma\left(\kappa-\frac{1}{2}\right) \Gamma\left(\frac{3}{2}\right)}\left(1+\frac{v^{2}}{\kappa v_{\mathrm{th}}^{2}}\right)^{-\kappa-1},
$$

where $v_{\text {th }}^{2}=\left(\frac{2 \kappa-3}{\kappa}\right) \frac{k T}{m}$ is the thermal velocity of the particles, $m$ is the mass of the particles of species, $n$ their number density, $T$ their equivalent temperature, and $\Gamma$ is the Gamma function. The free parameter $\kappa(\kappa>3 / 2)$ describes the deviation of the turbulent nonthermal distribution function from the Boltzmann distribution and is responsible for the shape of the distribution function and hence the slope of the energy spectrum of the suprathermal particles forming the tail of the velocity distribution function. A low value of $\kappa$ describes a power-law distribution with a strongly accelerated and hard spectrum collisionless plasma. For the large values $\kappa$, the function degenerates into a Maxwellian. Such deviations from Maxwellian distributions are expected to exist in low-density plasma where binary collisions are sufficiently rare.

In natural (space and astrophysical) or fusion plasmas, non-Maxwellian distributions originate from wave-plasma interactions, runaway electrons, turbulence, and the fusion of Alpha particles. Suprathermal radiation field and highly energetic beam sources are used for heating. Super-diffusion processes have been proposed to explain the suprathermal tails observed in many space plasmas (Treumann, 1997).

The presence of a high-energy tail component in a kappa distribution effectively changes the rate of resonant energy transfer between particles and plasma waves or by the action of plasma wave fluctuations in the process of transferring free energy to the wave and hence generating instabilities (Lazar et al., 2011). Wave absorption can increase or decrease with respect to the equivalent Maxwellian distribution function. In addition, plasma stability can either be enhanced or degraded.

The dispersion properties of low-frequency waves in a magnetized plasma by using kappa distribution has been derived by a number of authors. For instance, Rubab et al. (2009, 2010, 2011) employed kappa distribution to study kinetic Alfvén waves by showing that the cross field instability is found to be insensitive to the form of distribution function, while for parallel propagation $\left(V_{0} \| B_{0}\right)$ has a strong influence on the growth rates, i.e., for low values of kappa, the instability reduces, while by increasing the spectral index $(\kappa>5)$, the amplitude of the unstable region increases. They also showed that the parallel phase velocity of the dispersion relation remains unchanged, while nonthermality is found to be more effective in the perpendicular direction, which can be shown from the following relation:

$\omega^{2}=k_{\|}^{2} V_{A}^{2}\left[1+\frac{2 \kappa-3}{2 \kappa-1} k_{\perp}^{2} \rho_{\mathrm{d}}^{2} \pm i \pi n_{\mathrm{d} 0} \frac{r_{\mathrm{d}}^{2}}{k_{\|}}\right]$.

The same has been investigated by Watt and Rankin (2007) by showing that the parallel electron acceleration due to inertial Alfvén wave remains insensitive to parallel phase velocity by using kappa distribution and enumerated that wave particle interactions are enhanced, indicating that Landau damping increases for suprathermal particles. Furthermore, electron energy flux is also found to be dependent on kappa (Pierrard et al., 2001). Kinetic Alfvén solitary waves in a double layer structure have been studied by Gogoi and Khan (2010) using the kappa distribution function and it is found that the spectral index plays a significant role in determining the shape and size of solitons in double layers.

Observational data from the FREJA wave experiment showed that the Earth auroral plasma energization observed nonlinear soliton-type structures, which can be interpreted as solitary kinetic Alfvén waves (Wahlund et al., 1994; Louarn et al., 1994). Further, the particle distribution function in the auroral source regions with high-energy tails $\kappa<7$ has been observed (Christon et al., 1988; Kletzing et al., 2003). On the basis of theory and observations the data of solar winds suggest that such distributions give best fit curves. The existence of suprathermal distributions of charged particles at different heliospheric distances in the solar wind and in other space and planetary environments has been regularly confirmed by Interball/Aurora probes (Ermakova and Antonova, 
2006), Helios, (Prested et al., 2008) Ulysses (Zouganelis, 2008), Cassini (Schippers et al., 2008), and Wind observations (Salem et al., 2006). The presence of suprathermal charged particles has been vindicated in the magnetosphere of other planets such as Jupiter's megnetosheath (Krimigis et al., 1981; Mauk et al., 2004), Mercury (Christon, 1987), Neptune (Krimigis et al., 1989), and Saturn (Sittler Jr. et al., 1983). Rudakov et al. (2011) showed that the non-Maxwellian distribution function has a significant effect on the dynamics of solar wind plasmas. In particular, they showed that linear Landau damping leads to the formation of a plateau in the parallel electron distribution function, which diminishes the Landau damping rate significantly, and generalized nonlinear scattering of waves by plasma particles to short wavelengths. The incorporation of these effects leads to the steepening of the wave spectrum between the inertial and the dissipation ranges with a spectral index between 2 and 3 , as observed in the solar wind plasmas.

\subsection{Alfvén chaos}

Nonlinear dust Alfvén waves were investigated by Verheest (1994), where the dust dynamics is modeled by a number of cold, highly negatively charged and very massive fluids, in addition to electrons and protons; he showed that several low-frequency modes occur that are typical for the dust components, some of them described by the derivative nonlinear Schrödinger equation (DNLS) for electromagnetic waves. Verheest and Meuris (1996) showed that fluctuations in the charges of dust grains, due to capture or liberation of electrons and protons, induce density and momentum losses or gains in dusty plasmas, giving rise to wave damping or growth; in an intermediate regime the dust charging influences the nonlinear development of dust Alfvén waves, leading to a DNLS with a source term. Shukla and Verheest (2003) showed that nonlinear dynamics of modulated dust Alfvén wave packets is governed by a DNLS that admits localized envelope solitons; for dusty plasma parameters relevant to interstellar molecular clouds, the soliton width is on the order of a fraction of a parsec. Verheest (2004) applied the reductive perturbation theory to derive a DNLS to describe the nonlinear evolution of parallel propagating electromagnetic waves in multispecies plasmas; he showed that the DNLS cannot have stationary solitary waves, only envelope solitons. Verheest and Cattaert (2004) derived a DNLS for parallel electromagnetic modes in plasmas containing polydisperse charged dust; they showed that when modeling the charged dust by a power-law distribution in planetary rings and other astrophysical systems, it depends very much on the power-law index whether the smaller or the larger grains are more important. Pandey et al. (2008) studied the nonlinear wave propagation in collisional dusty plasma when electrons and ions are highly magnetized so that the Lorentz force acting on the plasma particles dominates its collision with the dust and charged dust remains weakly magnetized; they showed that large-amplitude waves can be easily excited in such a collisional dusty medium and can be described by the DNLS, whose soliton solutions explain the parsec scale structures in astrophysical plasmas.

The nonlinear evolution of a large-amplitude Alfvén wave propagating along an ambient magnetic field in the $x$ direction is described by the following derivative nonlinear Schrödinger equation (Hada et al., 1990; Chian et al., 1998, 2006)

$\partial_{t} b+\alpha \partial_{x}\left(|b|^{2} b\right)+i(\mu+i \eta) \partial_{x}^{2} b=S(x, t)$,

where $b=b_{y}+i b_{z}$ is the complex transverse wave magnetic field normalized to the constant ambient magnetic field $B_{0}$, $\eta$ is a dissipative scale length, time $t$ is normalized to the inverse of the ion cyclotron frequency $\omega_{\mathrm{ci}}=e B_{0} / m_{\mathrm{i}}$, the space coordinate $x$ is normalized to $c_{A} / \omega_{\mathrm{ci}}, c_{A}=B_{0} /\left(\mu_{0} \rho_{0}\right)^{1 / 2}$ is the Alfvén velocity, $\alpha=1 /[4(1-\beta)], \beta=c_{S}^{2} / c_{A}^{2}, c_{S}=$ $\left(P_{0} / \gamma \rho_{0}\right)^{1 / 2}$ is the acoustic velocity and $\mu$ is the dispersive parameter. The external driving force $S(x, t)=A \exp (i k \phi)$ is a monochromatic left-hand circularly polarized wave with a wave phase $\phi=x-V t$, where $V$ is a constant wave velocity, and $A$ and $k$ are arbitrary constants.

Low-dimensional chaos of DNLS has been studied by Hada et al. (1990), Buti (1997), Chian et al. (1998, 2006, 2007) and Rempel et al. (2007) by carrying out a dynamical analysis of the stationary solutions of Eq. (8) in the driver frame. This system exhibits a wealth of information on the nonlinear dynamics of Alfvén waves such as the routes from order to chaos via local (period-doubling and saddlenode) bifurcations and global bifurcations (interior crisis and boundary crisis). Although Eq. (8) was derived for a twofluid plasma, its mathematical structure is very similar to the DNLS in dusty plasmas. Hence, the results of chaos in DNLS in two-fluid plasmas are relevant for DNLS in dusty plasmas. Buti (1997) demonstrated that even a small fraction of charged dust grains can suppress chaos in Alfvén systems that are chaotic in the absence of dust particles. Note, however, that this analysis was performed in the absence of dissipation and noise. Since a dynamical system is sensitive to the variation of the physical parameters, the presence of dissipation and noise may alter the result of Buti (1997) significantly.

The effects of noise on Alfvén chaos were investigated by Rempel et al. (2006, 2008). By adding a Gaussian noise to the stationary solutions of the DNLS, Rempel et al. (2006) studied the occurrence of extrinsic transients and attractor hopping in a multistable regime of Alfvén waves. The complex dynamics of Alfvén waves described by DNLS was studied by Rempel et al. (2008) when a nonlinear Alfvén wave is driven towards an intermittent regime by the addition of noise. The effects of Gaussian and non-Gaussian noise were compared. In the intermittent regime, a nonlinear Alfvén wave exhibits random qualitative changes in its dynamics as the result of competition between three co-existing attractors and a chaotic saddle embedded in the fractal basin boundary. 
Low-dimensional chaos of DNLS can also be studied by considering Alfvén modulational wave coupling processes (Sánchez-Arriaga et al., 2009; Miranda et al., 2012). Sánchez-Arriaga et al. (2009) studied a truncation model of DNLS with three resonant traveling waves. They showed that the modulational instability criterion is in agreement with the existence of two hyperbolic fixed points joined by a heteroclinic orbit that forces the exchange of energy among the three waves. In the presence of dissipative and growth terms, the system exhibits very complex dynamics including multi-attractors and period-doubling bifurcation to chaos. Miranda et al. (2012) studied the truncated three-wave model of DNLS involving a linearly unstable Alfvén wave and two linearly damped Alfvén waves with different damping rates. The maximum Lyapunov exponent was computed as a function of the damping rates in a two-parameter phase space, and shrimp-shaped self-similar structures in the parameter phase space were identified. By varying the damping rate of the low-frequency wave, a bifurcation diagram was constructed to show saddle-node bifurcation and interior crisis associated with a periodic window. Chaotic saddles and their stable and unstable manifolds were detected, and it was shown that the interaction between two chaotic saddles via the coupling of unstable periodic orbits can lead to crisis-induced intermittency.

The aforementioned studies on Alfvén chaos elucidate how Alfvén intermittency appears in space and astrophysical plasmas, such as the magnetic intermittent turbulence observed in the solar wind (Koga et al., 2007; Sahraoui, 2008; Chian and Miranda, 2009; Chian and Muñoz, 2011), due to nonlinear dynamical phenomena such as the type-I intermittency induced by a saddle-node bifurcation, the crisisinduced intermittency induced by an interior crisis, and the extrinsic intermittency induced by a noise (Hada et al., 1990; Chian et al., 1998, 2006, 2007; Rempel et al., 2006, 2008; Sánchez-Arriaga et al., 2009; Miranda et al., 2012).

\section{Alfvén waves: space plasma applications}

Alfvén waves are responsible for a variety of physical processes in space plasmas as described in Sect. 2. Hence space plasmas provide an ideal laboratory to study the properties of this mode. As an illustration, we review briefly some observations of Alfvén waves in the Earth's auroral region.

Kinetic Alfvén waves play an important role in auroral particle acceleration (Chaston et al., 2000, 2006). Field aligned currents (FACs) are the major source of ionospheremagnetosphere coupling and the formation of auroras, as the change in the auroras is associated with the change in the auroral currents. The auroral acceleration not only occurs due to the closure of FACs (Paschmann et al., 2003), but also in the case of empowering Alfvénic auroras through the action of free energy on a small scale (Chaston et al., 2008).
Chaston and Seki (2010) simulated a 3-D model to structure small-scale auroras by considering two different scenarios, where at the electron inertial scale slippage between plasma and geomagnetic field lines becomes significant, leading the thin auroral sheet towards Kelvin-Helmholtz instability and hence the generation of vortices, while the other setup involves a resistive layer between ionosphere and magnetosphere.

Measurements from the FAST satellite (Chaston et al., 2000, 2006) and the POLAR spacecraft (Wygant et al., 2000) have speculated the existence of small-scale Alfvén waves that carry a large net Poynting flux along magnetic field lines towards the Earth. These are the intense and shortlived bursts of precipitating suprathermal electron flux $(E<$ $1 \mathrm{keV}$ ). Chaston et al. (2003) developed numerical simulations that confirmed that the downward energy flux of electrons accelerated by inertial Alfvén waves is responsible for exciting visible auroras.

The phenomenon associated with the Alfvén waves in auroral region can also be examined by in situ measurements where the fluctuating electric and magnetic fields and their correlation for the propagation of Alfvén waves can be determined. The parallel current that closes in the ionosphere is expected to be due to these fields in quasi-static structures. The electrons accelerated through such structures, where the field line potential is small as compared to the variation in potential, are shifted in energy, which show first a maximum in energy spectrum and then a decrease, following an invertedV feature (Frank and Ackerson, 1972). It has been shown by Stasiewicz et al. (2000) that for quasi-static auroral arclike structures, the magnetic field has a direct relation to field aligned currents, given by

$$
\frac{1}{\mu_{0}} \frac{\partial b_{\perp}}{\partial s}=J_{\perp}=\sigma_{\mathrm{p}} E_{\perp},
$$

where $\sigma_{\mathrm{p}}$ is the Pedersen conductivity in the ionosphere.

Reimei spacecraft observations by Asamura et al. (2009) revealed spatial-temporal evolution of small-scale Alfvén wave generation in auroral acceleration regions. They observed the dispersive electron burst, auroral structures driven by inverted- $V$ electrons and the distribution function responsible for driving them. They concluded that inverted-V electron population is responsible for auroral luminosity, while low-energy electrons assemble information from the regions where inverted- $\mathrm{V}$ events take place.

Observational data from the FREJA wave experiment showed a close connection between Alfvén and ion acoustic wave activity within auroral energization regions. Wahlund et al. (1994) found Alfvénic signatures that appear more electrostatic-like. The waveforms contain features with scales on the order of the ion gyroradius where the kinetic (thermal) effects are expected to play a role in highfrequency electrostatic turbulence up to $15 \mathrm{kHz}$. They also observed that the transverse scale of the spikes is on the order of the ion inertial scale that is comparable to a discrete 
auroral arc and showed that the Earth auroral plasma energization is associated with nonlinear soliton-type structures that can be interpreted as solitary kinetic Alfvén waves. Based on this data a number of authors identified electromagnetic waveforms having characteristics of Alfvén waves and suprathermal electron bursts (Wahlund et al., 1998; Gary et al., 1998).

The particle distribution function in the auroral source regions with high-energy tails $(\kappa<7)$ is also observed (Christon et al., 1988; Kletzing et al., 2003); therefore, it may be useful to study the Lorentzian inertial/kinetic Alfvén waves in this region.

\section{Alfvén waves: astrophysical plasma applications}

In this section we present models/results related to Alfvén wave dynamics in stellar winds and star formation where dust grains are embedded.

\subsection{Stellar winds}

Alfvén waves might play an important role in driving stellar winds in many regions of the HR diagram. For cool giant and supergiant stars, the most promising mechanism is an outward directed flux of Alfvén waves. This mechanism can explain both the high mass-loss rate of $\sim 10^{-8}-10^{-6} \mathrm{M}_{\odot} \mathrm{yr}^{-1}$ and the low terminal velocities of $\sim 20-80 \mathrm{~km} \mathrm{~s}^{-1}$ of these winds. It is a characteristic of cool winds to have terminal velocities $u_{\infty}$ less than the escape velocity at the stellar surface, $v_{\mathrm{e} 0}$, and the observations show that $u_{\infty} \sim v_{\mathrm{e} 0} / 2$. The waves can produce significant mass loss even in stars that do not have hot coronas or strong radiative fluxes. This treatment was previously developed by Hollweg (1973); Hartmann and MacGregor (1980); Hartmann and Avrett (1984); JatencoPereira and Opher (1989), for example.

In the solar corona the presence of Alfvén waves was confirmed observationally (Tomczyk et al., 2007). There is direct evidence for Alfvén waves in the solar wind: waves are observed as large perturbations in the magnetic field and as negligible perturbations in density (Chian et al., 1998, 2006), and if there are oscillations in the magnetic field at the base of the wind, Alfvén waves will be generated. These waves propagate outward, dissipate their energy and transfer the momentum to accelerate the wind. It is known that the coronal holes are the source of the high-speed solar wind streams at the Earth's orbit. The measurement of particle fluxes mapped back to coronal holes implies mass fluxes at the base of a coronal hole that are 5-10 times greater than the mass flux obtained by assuming a spherically symmetric solar wind (Hundhausen, 1977). For coronal holes in the Sun, the solid angle for a radial geometry, $\Omega_{0}$, is observed to suffer an expansion of from $\Omega_{0}$ at the surface (i.e., at $r=R_{\odot}$ ) to $\sim 7 \Omega_{0}$ at $r=3 R_{\odot}$ (Munro and Jackson, 1977). Beyond $3 R_{\odot}$, the edges of the coronal hole are radial and a net non-radial expansion factor $F=\Omega / \Omega_{0}=7.26$ is found. For cool giant and supergiant stars it is assumed that $F=10$ (JatencoPereira and Opher, 1989).

It was shown by Cramer et al. (2002) that the dispersion relation of Alfvén waves is modified in a dusty plasma, because charged grains in a magnetized plasma are highly coupled to the waves due to cyclotron resonances. Since it is observed that the winds of late-type supergiant stars contain significant amounts of dust, the Alfvén waves can be dissipated in these winds. In order to evaluate how this damping mechanism can accelerate the wind, Vidotto and Jatenco-Pereira (2006) proposed a model that considers an outward flux of Alfvén waves generated at the stellar surface that is damped only due to the interaction with dust grains. They studied a K5 supergiant star with the following magnetic field configuration: from the surface, the magnetic field configuration is divergent and after a transition radius $r_{\mathrm{t}}$, it becomes radial. If $A(r)$ is the cross section of the geometry at a radial distance $r$, then

$A(r)= \begin{cases}A\left(r_{0}\right)\left(r / r_{0}\right)^{S} & \text { if } r \leq r_{\mathrm{t}} \\ A\left(r_{0}\right)\left(r_{\mathrm{t}} / r_{0}\right)^{S}\left(r / r_{\mathrm{t}}\right)^{2} & \text { if } r>r_{\mathrm{t}},\end{cases}$

where $S$ is a parameter that determines the divergence of the geometry. The mass, energy, and momentum equations, which were solved simultaneously by taking into account both the radiative losses and the wave heating in the energy equation, determine the temperature.

Vidotto and Jatenco-Pereira (2006) assumed that the wave energy density is dissipated as follows:

$\epsilon(\omega)=\epsilon_{0}(\omega) \frac{M_{0}}{M}\left(\frac{1+M_{0}}{1+M}\right)^{2} \exp \left\{-\int_{r_{0}}^{r} \frac{1}{L} \mathrm{~d} r^{\prime}\right\}$,

where $M_{0}=u_{0} / v_{\mathrm{A} 0}$ is the initial Alfvén-Mach number, $M=u / v_{A}$ is the Alfvén-Mach number, the Alfvén speed is given by $v_{A}=(B / \sqrt{4 \pi \rho})$, and $L$ is the damping length. They also assumed that $\epsilon_{0}(\omega)$, the initial wave energy density, obeys a power law as in the Sun (Tu et al., 1989)

$\epsilon_{0}(\omega)=\epsilon_{0}\left(\omega_{0}\right)\left(\frac{\omega}{\omega_{0}}\right)^{-0.6}$,

and the following initial parameters for a K5 supergiant star: $r_{0}=400 \mathrm{R}_{\odot}, M_{\star}=16 \mathrm{M}_{\odot}$, initial Alfvén wave flux $\phi_{A_{0}}=5.5 \times 10^{6} \mathrm{erg} \mathrm{cm}^{-2} \mathrm{~s}^{-1}, \rho_{0}=1.07 \times 10^{-13} \mathrm{~g} \mathrm{~cm}^{-3}$, $B_{0}=10 \mathrm{G}, T_{0}=3500 \mathrm{~K}$, and $S=5.0$. They also considered a distribution function of dust grain sizes, as first proposed by Mathis et al. (1977) for grains in the interstellar medium

$f(a)=\frac{p-1}{1-a_{\mathrm{m}}^{1-p}} a^{-p}$,

where $a_{\mathrm{m}}=a_{2} / a_{1}$ with $a_{1}$ being the minimum dust grain radius, and $a_{2}$ the maximum dust grain radius. $a_{1}=5.0 \times 10^{-7}$ 
and $a_{2}=2.5 \times 10^{-5} \mathrm{~cm}$ (Rodgers and Glassgold, 1991) and $p=4$ were adopted.

Vidotto and Jatenco-Pereira (2006) obtained the wind velocity and temperature profiles of a K5 supergiant star compatible with the observations (e.g., the terminal velocity lower than the surface escape velocity and a rise in the wind temperature as the distance increases from the stellar surface similar to the solar wind), which shows that the dustcyclotron damping mechanism can be very promising in order to accelerate the wind.

\subsection{Star formation regions}

The literature contains numerous studies comprising theoretical and numerical simulations and concerning discs around the young stellar objects. Usually the central object provides all of the gravitational force on the disc and the orbit of the material in the disc is close to circular (Keplerian) and generally characterized as differential. The ambient of the cloud that gives rise to the process of star formation contains turbulence, magnetic field and dust that must be present in the later stages of pre-main-sequence stellar evolution (e.g., Schulz, 2005).

The magneto-rotational instability (MRI, Balbus and Hawley, 1991) is probably the mechanism responsible for magneto-hydrodynamic (MHD) turbulence that leads to disc accretion: it provides a necessary torque for the disc's particles to lose angular momentum and to move towards the central object. The concept of a quiescent layer incapable of allowing the operation of the MRI in a protostar disc was proposed by Gammie (1996). In this dead zone, the disc is not sufficiently ionized, making it difficult for the gas to be magnetically coupled to the field lines. However, if the ionization degree is sufficiently high, the MRI can operate and the disc becomes turbulent (Adams, 2010).

In a series of papers, Turner et al. (2007) and Turner and Sano (2008) used the minimum-mass solar nebula as the disc model to study under which conditions the dead zone can be eradicated, leaving the entire disc active. They showed that magnetic stresses can occur in the dead zone and that the interior of the disc retains a weak coupling to the magnetic field. Jatenco-Pereira (2013) investigated the damping of Alfvén waves as an extra non-thermal source of heating that could increase the midplane disc temperature. Although this non-thermal heating mechanism had already been suggested by Vasconcelos et al. (2000), it was not considered in the context when the dust particles are taken into account in the plasma. The waves are damped while propagating through the disc along the magnetic field lines that are taken to be perpendicular to the disc midplane. While they propagate, the waves are damped and transfer energy to the disc (Jatenco-Pereira, 2013).

Jatenco-Pereira (2013) assumed that the disc is geometrically thin, optically thick, and is in the Keplerian rotation while adopting the Shakura and Sunyaev (1973) model with
Table 1. Effective temperature $T_{\text {eff }}$ of the disk as a function of the parameterized initial flux of Alfvén waves $\left(\Phi_{A}=f^{2} v_{A}^{3} \rho\right)$.

\begin{tabular}{cccc}
\hline Model & $f$ & $T_{\text {eff }}(r=0.1 \mathrm{AU})$ & $T_{\text {eff }}(r=1 \mathrm{AU})$ \\
$(\mathrm{K})$ & $(\mathrm{K})$
\end{tabular}

some changes proper for a cold ambient: the radiation pressure term in the equation of state was neglected and the opacity was given by Bell and Lin (1994). The $\alpha$ prescription (Shakura and Sunyaev, 1973) suggests that the viscosity is set by the interaction of turbulent eddies: the maximum size of a turbulent cell cannot exceed the disc scale height $(H)$ and its velocity is less than the sound speed $\left(c_{\mathrm{s}}\right)$. Hence the maximum kinematic viscosity is then $v_{\max }=H c_{\mathrm{s}}$. It is considered that when a disc is irradiating like a black body, its effective temperature is obtained by setting the equilibrium between the energy flux liberated by the viscous dissipation $\left(\mathcal{F}_{\mathrm{v}}\right)$ and the black-body energy flux $\left(\sigma T_{\text {eff }}^{4}\right)$. When an additional source of energy for the discs is included, the equilibrium between the energy gained and the energy dissipated (black body) is given by Vasconcelos et al. (2000)

$\mathcal{F}_{\mathrm{V}}(H)+\mathcal{F}_{A}(H)=\sigma T_{\text {eff }}^{4}$,

where $\mathcal{F}_{A}(H)$ is the energy flux dissipated by the Alfvén waves. It was assumed that the wave energy flux per frequency range can be described by a power law as in Sect. 4.1.

The results for a typical protostar are characterized by $M_{\star}=0.7 M_{\odot}$ and $R_{\star}=2.5 R_{\odot}$ and the accretion disc with a mean molecular weight $\mu=2.33$, internal radius $R_{\mathrm{i}}=2 R_{\star}$, accretion rate $\dot{M}=10^{-7} \mathrm{M}_{\odot} \mathrm{yr}^{-1}, \alpha=10^{-2}$, the minimum and maximum grain sizes are $a_{\min }=0.001 \mu \mathrm{m}$ and $a_{\max }=$ $0.25 \mu \mathrm{m}$, respectively, of the effective temperature $T_{\text {eff }}$ for two different locations of the disk as a function of the initial flux of Alfvén waves, parameterized by $f\left(\Phi_{A}=f^{2} v_{A}^{3} \rho\right)$ compared to a purely $\alpha$ model are shown in Table 1 .

We show that the damping of Alfvén waves by the dust cyclotron mechanism is more efficient than the "anomalous" viscosity mechanism and can increase the effective temperature of the disc, allowing the MRI to take place in a large part of the disc (Jatenco-Pereira, 2013).

\section{Summary}

We reviewed Alfvén waves in space and astrophysical dusty plasmas by introducing some basic physical concepts of the dusty environments. It was shown that the dispersion relation of Alfvén waves is modified significantly in a dusty plasma since the charged grains in a magnetized plasma are highly coupled to the waves due to cyclotron resonances. The charged dust particles introduce a cutoff in the Alfvén 
wave at the dust cyclotron frequency. If a distribution of grain sizes is considered, a large band of resonance frequencies appear instead of a single frequency. We discussed the damping of kinetic Alfvén waves, the Lorentzian particle velocity distribution functions, and the nonlinear evolution of large-amplitude Alfvén waves to chaos. We also reviewed some applications in space and astrophysical plasmas, showing the importance of Alfvén waves in these studies. Finally we would like to add some future issues that we intend to develop: (i) the role of damping of KAWs in coronal holes and the consequences for solar wind acceleration; (ii) the study of Lorentzian inertial/kinetic Alfvén waves in aurora regions to elucidate the role of particle distribution function; (iii) the contribution of damping of KAWs for acceleration of stellar winds, among other studies.

Acknowledgements. VJP would like to thank the Brazilian agency CNPq (under grant 304184/2010-1) for partial support. ACLC thanks CNPq for support, the European Commission for the award of a Marie Curie International Incoming Fellowship, and the Paris Observatory for hospitality.

Edited by: T. Hada

Reviewed by: two anonymous referees

\section{References}

Adams, F. C.: The birth environment of the solar system, Annu. Rev. Astron. Astr., 48, 47-85, doi:10.1146/annurev-astro-081309130830, 2010.

Asamura, K., Chaston, C. C., Itoh, Y., Fujimoto, M., Sakanoi, T., Ebihara, Y., Yamazaki, A., Hirahara, M., Seki, K., Kasaba, Y., and Okada, M.: Sheared flows and samll-scale Alfvén wave generation in the auroral acceleration region, Geophys. Res. Lett., 36, L05105, doi:10.1029/2008GL036803, 2009.

Balbus, S. A. and Hawley, J. F.: A powerful local shear instability in weakly magnetized disks. I. Linear analysis, Astrophys. J., 376, 214-222, 1991.

Bell, K. R. and Lin, D. N. C.: Using FU Orionis outbursts to constrain self-regulated protostellar disk models, Astrophys. J., 427, 987-1004, 1994.

Buti, B.: Control of chaos in dusty plasmas, Phys. Lett. A, 235, 241-247, 1997.

Chaston, C. C. and Seki, K.: Small-scale auroral current sheet structuring, J. Geophys. Res., 115, A11221, doi:10.1029/2010JA015536, 2010.

Chaston, C. C., Carlson, C. W., Ergun, R. E., and McFadden, J. P.: Alfvén Waves, Density Cavities and Electron Acceleration Observed from the FAST Spacecraft, Phys. Scripta, T84, 64-68, 2000.

Chaston, C. C., Peticolas, L. M., Bonnell, J. W., Carlson, C. W., Ergun, R. E., McFadden, J. P., and Strangeway, R. J.: Width and brightness of auroral arcs driven by inertial Alfvén waves, J. Geophys. Res., 108, 1091, doi:10.1029/2001JA007537, 2003.

Chaston, C. C., Genot, V., Bonnell, J. W., Carlson, C. W., McFadden, J. P., Ergun, R. E., Strangeway, R. J., Lund, E. J., and
Hwang, K. J.: Ionospheric erosion by Alfvén waves, J. Geophys. Res., 111, A03206, doi:10.1029/2005JA011367, 2006.

Chaston, C. C., Salem, C., Bonnell, J. W., Carlson, C. W., Ergun, R. E., Strangeway, R. J., and McFadden, J. P.: The turbulent Alfvénic aurora, Phys. Rev. Lett., 100, 175003, doi:10.1103/PhysRevLett.100.175003, 2008.

Chian, A. C.-L. and Miranda, R. A.: Cluster and ACE observations of phase synchronization in intermittent magnetic field turbulence: a comparative study of shocked and unshocked solar wind, Ann. Geophys., 27, 1789-1801, doi:10.5194/angeo-271789-2009, 2009.

Chian, A. C.-L. and Muñoz, P. R.: Detection of current sheets and magnetic reconnections at the turbulent leading edge of interplanetary coronal mass ejection, Astrophys. J. Lett., 733, L34, doi:10.1088/2041-8205/733/2/L34, 2011.

Chian, A. C.-L., Borotto, F. A., and Gonzalez, W. D.: Alfvén intermittent turbulence driven by temporal chaos, Astrophys. J., 505, 993-998, 1998.

Chian, A. C.-L., Kamide, Y., Rempel, E. L., and Santana, W. M.: On the chaotic nature of solar-terrestrial environment: interplanetary Alfvén intermittency, J. Geophys. Res., 111, A07S03, doi:10.1029/2005JA011396, 2006.

Chian, A. C.-L., Santana, W. M., Rempel, E. L., Borotto, F. A., Hada, T., and Kamide, Y.: Chaos in driven Alfvén systems: unstable periodic orbits and chaotic saddles, Nonlin. Processes Geophys., 14, 17-29, doi:10.5194/npg-14-17-2007, 2007.

Christon, S. P.: A comparison of the Mercury and Earth magnetosphere measurements and substorm timescales, Icarus, 71, 448471, doi:10.1016/0019-1035(87)90040-6, 1987.

Christon, S. P., Mitchell, D., Williams, D., Frank, L., Huang, C., and Eastman, T.: Energy spectra of plasma sheet ions and electrons from about $50 \mathrm{eV} / \mathrm{e}$ to about $1 \mathrm{MeV}$ during plasma temperature transitions, J. Geophys. Res., 93, 2562-2572, 1988.

Collier, M. R.: On generating kappa-like distribution functions using velocity space Levy flights, Geophys. Res. Lett., 20, 15311534, 1993.

Cramer, N.: The Physics of Alfvén Waves, Wiley, Berlin, 2001.

Cramer, N., Verheest, F., and Vladimirov, S.: The Alfvén resonance in a dusty plasma with a distribution of grain sizes, Phys. Plasmas, 9, 4845-4850, 2002.

D'Angelo, N.: Coulomb solids and low-frequency fluctuations in RF dusty plasmas, J. Phys. D Appl. Phys., 28, 1009-1010, 1995.

Das, A. C., Misra, A. K., and Goswami, K. S.: Kinetic Alfvén wave in three-component dusty plasmas, Phys. Rev. E, 53, 4051-4055, 1996.

de Angelis, U.: The physics of dusty plasmas, Phys. Scripta, 45, 465-474, 1992.

de Juli, M. C., Schneider, R. S., Ziebell, L. F., and JatencoPereira, V.: Effects of dust-charge fluctuation on the damping of Alfvén waves in dusty plasmas, Phys. Plasmas, 12, 052109, doi:10.1063/1.1899647, 2005.

Elmegreen, B. G. and Fiebig, D.: On the minimum length for magnetic waves in molecular clouds, Astron. Astrophys. 270, 397400, 1993.

Ermakova, N. O. and Antonova, E. E.: On the role of nonMaxwellian forms of distribution functions in the process of acceleration of auroral particles, Int. Conf. Substorms, 8, 61-64, 2006. 
Frank, L. A. and Ackerson, K. L.: Local-time survey of plasma at low altitudes over the auroral zones, J. Geophys. Res., 77, 41164127, doi:10.1029/JA077i022p04116, 1972.

Gaelzer, R., de Juli, M. C., Schneider, R. S., and Ziebell, L. F.: Obliquely propagating Alfvén waves in a Maxwellian dusty plasma, Plasma Phys. Contr. F., 51, 015011, doi:10.1088/07413335/51/1/015011, 2009.

Gaelzer, R., de Juli, M. C., and Ziebell, L. F.: Effect of superthermal electrons on Alfvén wave propagation in the dusty plasmas of solar and stellar winds, J. Geophys. Res., 115, A09109, doi:10.1029/2009JA015217, 2010.

Gammie, C. F.: Layered accretion in T Tauri disks, Astrophys. J., 457, 355-362, 1996.

Gary, J. B., Zanetti, L. J., Anderson, B. J., Potemra, T. A., Clemmons, J. H., Winningham, J. D., and Sharber, J. R.: Identification of auroral oval boundaries from in situ magnetic field measurements, J. Geophys. Res., 103, 4187-4197, doi:10.1029/97JA02395, 1998.

Gary, S. P. and Borovsky, J. E.: Alfvén-cyclotron fluctuations: Linear Vlasov theory, J. Geophys. Res., 109, A06105, doi:10.1029/2004JA010399, 2004.

Goertz, C.: Dusty plasmas in the solar system, Rev. Geophys., 27, 271-292, 1989.

Gogoi, R. and Khan, M.: Arbitrary amplitude kinetic Alfvén solitary waves and double layers in a non Maxwellian plasma, Phys. Plasmas, 17, 112311, doi:10.1063/1.3506820, 2010.

Hada, T., Kennel, C. F., Buti, B., and Mjølhus, E.: Chaos in driven Alfvén systems, Phys. Fluids B, 2, 2581-2590, 1990.

Hartmann, L. and Avrett, E. H.: On the extended chromosphere of Alpha Orionis, Astrophys. J., 284, 238-249, 1984.

Hartmann, L. and MacGregor, K. B.: Momentum and energy deposition in late-type stellar atmospheres and winds, Astrophys. J., 242, 260-282, 1980

Hasegawa, A. and Cheng, L.: Kinetic processes in plasma heating by resonant mode conversion of Alfvén wave, Phys. Fluids, 19, 1924-1932, 1976.

Hollweg, J. V.: Alfvén waves in a two-fluid model of the solar wind, Astrophys. J., 181, 547-566, 1973.

Hundhausen, A.: Plasma Flow from the Sun, in: The Solar Output and its Variation, Proceedings, edited by: White, O. R., Colorado Associated University Press, Boulder, USA, 36 pp., 1977.

Jatenco-Pereira, V.: Damping of Alfvén waves as a heating source in protostellar accretion discs, Mon. Not. R. Astron. Soc., 431, 3150-3158, 2013.

Jatenco-Pereira, V. and Opher, R.: Effect of diverging magnetic fields on mass loss in late-type giant stars, Astron. Astrophys., 209, 327-336, 1989.

Kamaya, H. and Nishi, R.: Frozen condition for the charged particles in molecular clouds, Astrophys. J., 543, 257-270, 2000.

Keidar, M., Shashurin, A., Volotskova, O., Stepp, M. A., Srinivasan, P., Sandler, A., and Trink, B.: Cold atmospheric plasma in cancer therapy, Phys. Plasmas, 20, 057101, doi:10.1063/1.4801516, 2013.

Khan, M. S. A., Sultana, S., and Samilullah, M.: Nature of electromagnetic wave in uniform dusty plasma, International Journal of Science and Research (IJSR), 2, 132-136, 2013.

Kletzing, C. A., Scudder, J. D., Dors, E. E., and Curto, C.: Auroral source region: Plasma properties of the high-latitude plasma sheet, J. Geophys. Res., 108, 1360, doi:10.1029/2002JA009678, 2003.

Koga, D., Chian, A. C.-L., Miranda, R. M., and Rempel, E. L.: Intermittent nature of solar wind turbulence near the Earth's bow shock: Phase coherence and non-Gaussianity, Phys. Rev. E, 75, 046401, doi:10.1103/PhysRevE.75.046401, 2007.

Köritzer, J., Boxhammer, V., Schafer, A., Shimizu, T., Klämptl, T. G., Li, Y.-F., Welz, C., Schwenk-Zieger, S., Morfill, G. E., Zimmermann, J. L., and Schlegl, J.: Restoration of sensitivity in chemo - resistant glioma cells by cold atmospheric plasma, PLoS ONE, 8, e64498, doi:10.1371/journal.pone.0064498, 2013.

Krimigis, S. M., Carbary, J. F., Keath, E. P., Bostrom, C. O., Axford, W. I., Gloeckler, G., Lanzerotti L. J., and Armstrong, T. P.: Characteristics of hot plasma in the Jovian magnetosphere: Results from Voyager spacecraft, J. Geophys. Res., 86, 8227-8257, doi:10.1029/JA086iA10p08227, 1981.

Krimigis, S. M., Armstrong, T. P., Axford, W. I., Bostrom, C. O., Cheng, A. F., Gloeckler, G., Hamilton, D. C., Keath, E. P., Lanzerotti, L. J., Mauk, B. H., and Van Allen, J. A.: Hot plasma and energetic Particles in Neptune's Magnetosphere, Science, 246, 4936, doi:10.1126/science.246.4936.1483, 1989.

Lazar, M., Poedts, S., and Schlickeiser, R.: Instability of the parallel electromagnetic modes in Kappa distributed plasmas - I. Electron whistler-cyclotron modes, Mon. Not. R. Astron. Soc., 410, 663-670, doi:10.1111/j.1365-2966.2010.17472.x, 2011.

Louarn, J. E., Wahlund, T., Chust, H., de Feraudy, A., Roux, B., Holback, P. O., Dovnor, A., Ericksson, I., and Holmgren, G.: Observation of kinetic Alfvén waves by the FREJA spacecraft, Geophys. Res. Lett., 21, 1847-1850, 1994.

Mathis, J., Rumple, W., and Nordsiek, K.: The size distribution of interstellar grains, Astrophys. J., 217, 425-433, 1977.

Mauk, B. H., Mitchell, D. G., McEntire, R. W., Paranicas, C. P., Roelof, E. C., Williams, D. J., Krimigis, S. M., and Lagg, A.: Energetic ion characteristics and neutral gas interactions in Jupiter's magnetosphere, J. Geophys. Res., 109, A09S12, doi:10.1029/2003JA010270, 2004.

Miranda, R. A., Rempel, E. L., and Chian, A. C.-L.: Chaotic saddles in nonlinear modulational interactions in a plasma, Phys. Plasmas, 19, 112303, doi:10.1063/1.4766472, 2012.

Munro, R. H. and Jackson, B. V.: Physical properties of a polar coronal hole from 2 to 5 solar radii, Astrophys. J., 213, 874-886, 1977.

Nakano, T., Nishi, R., and Umebayashi, T.: Mechanism of magnetic flux loss in molecular clouds, Astrophys. J., 573, 199-214, 2002.

Pandey, B. P., Vladimirov, S. V., and Samarian, A.: Nonlinear waves in collisional dusty plasma, Phys. Plasmas, 15, 053705, doi:10.1063/1.2918341, 2008.

Paschmann, G., Haaland, S., and Treumann, R.: Auroral Plasma Physics, Kluwer Academic, Dordrecht, p. 94, 2003.

Pierrard, V., Maksimovic, M., and Lemaire, J.: Core, Halo and Strahl Electrons in the Solar Wind, Astrophys. Space Sci., 277, 195-200, doi:10.1023/A:1012218600882, 2001.

Prested, C., Schwadron, N., Passuite, J., Randol, B., Stuart, B., Crew, G., Heerikhuisen, J., Pogorelov, N., Zank, G., Opher, M., Allegrini, F., cComas, M. Reno, D. J., Roelof, E., Fuselier, S., Funsten, H., Moebius, E., and Saul, L.: Implications of solar wind suprathermal tails for IBEX ENA images of the heliosheath, J. Geophys. Res., 113, A06102, doi:10.1029/2007JA012758, 2008. 
Rao, N. N.: Advances in dusty plasmas, edited by: Shukla, P. K., Mendis, D. A., Desai, T., World Scientific, Singapore, 1996.

Rao, N. N., Shukla, P. K., and Yu, M. Y.: Dust-acoustic waves in dusty plasmas, Planet. Space Sci., 38, 543-546, 1990.

Rempel, E. L., Wanderson, S. M., and Chian, A. C.-L.: Alfvén multistability: transient and intermittent dynamics induced by noise, Phys. Plasmas, 13, 032308, doi:10.1063/1.2186527, 2006.

Rempel, E. L., Chian, A. C.-L., and Miranda, R. A.: Chaotic saddles at the onset of intermittent spatiotemporal chaos, Phys. Rev. E, 76, 056217, doi:10.1103/PhysRevE.76.056217, 2007.

Rempel, E. L., Chian, A. C.-L., Koga, D., Miranda, R. A., and Santana, W. M.: Alfvén complexity, Int. J. Bifurcation Chaos, 18, 1697-1703, 2008.

Rodgers, B. and Glassgold, A. E.: The temperature of the circumstellar envelope of Alpha Orionis, Astrophys. J., 382, 606-616, 1991.

Rubab, N., Erkaev, N. V., and Biernat, H. K.: Dust kinetic Alfvén wave and acoustic waves in a Lorentzian plasma, Phys. Plasmas, 16, 103704, doi:10.1063/1.3244625, 2009.

Rubab, N., Erkaev, N. V., Langmayr, D., and Biernat, H. K.: Kinetic Alfvén wave instability in a Lorentzian dusty magnetosplasma, Phys. Plasmas, 17, 103704, doi:10.1063/1.3491336, 2010.

Rubab, N., Erkaev, N. V., Biernat, H., K., and Langmayr, D.: Kinetic Alfvén wave instability in a Lorentzian dusty plasma: Non-resonant particle approach, Phys. Plasmas, 18, 073701, doi:10.1063/1.3599600, 2011.

Rudakov, L., Mithaiwala, M., Ganguli G., Crabtree, C.: Linear and nonlinear Landau resonance of kinetic Alfvén waves: Consequences for electron distribution and wave spectrum in the solar wind, Phys. Plasmas, 18, 012307, doi:10.1063/1.3532819, 2011.

Sahraoui, F.: Diagnosis of magnetic structures and intermittency in space-plasma turbulence using the technique of surrogate data, Phys. Rev. E, 78, 026402, doi:10.1103/PhysRevE.78.026402, 2008.

Salem, C., Hubert, D., Bale, S. D., and Lin, R. P.: Nature and origin of the electron distribution functions in the slow and fast solar wind at $1 \mathrm{AU}$ : Wind observations, 36th COSPAR Scientific Assembly, Beijing, China, 16-23 July 2006.

Sánchez-Arriaga, G., Hada, T., and Nariyuki, Y.:, The truncation model of the derivative nonlinear Schrodinger equation, Phys. Plasmas, 16, 042302, doi:10.1063/1.3093383, 2009.

Schippers, P., Blanc, M., André, N., Dandouras, I., Lewis, G. R., Gilbert, L. K., Persoon, A. M., Krupp, N., Gurnett, D. A., Coates, A. J., Krimigis, S. M., Young, D. T., and Dougherty, M. K.: Multi-instrument analysis of electron populations in Saturn's magnetosphere, J. Geophys. Res., 113, A07208, doi:10.1029/2008JA013098, 2008.

Schulz, N. S.: From Dust To Stars, in: Studies of the Formation and Early Evolution of Stars, Springer Praxis Books in Astrophysics and Astronomy, Praxis Publishing Ltd, ISBN 3-54023711-9, 147 pp., 2005.

Sittler Jr., E. C., Ogilvie, K. W., and Scudder, J. D.: Survey of lowenergy plasma electrons in Saturn's magnetosphere: Voyagers 1 and 2, J. Geophys. Res., 88, 8847-8870, 1983.

Shakura, N. I. and Sunyaev, R. A.: Black holes in binary systems. Observational appearance, Astron. Astrophys., 24, 337355, 1973.
Shukla, P. K. and Verheest, F.: Dust Alfvén envelope solitons in astrophysical dusty plasmas, Astron. Astrophys., 401, 849-850, 2003.

Sorasio, G. and Rosenberg, M.: Instability of higher-harmonic electrostatic dust cyclotron waves, J. Plasma Phys., 65, 319-329, 2001.

Stasiewicz, K., Bellan, P., Chaston, C., Kletzing, C., Lysak, R., Maggs, J., Pokhotelov, O., Seyler, C., Shukla, P., Stenflo, L., Streltsov, A., and Wahlund, J.-E.: Small scale Alfvénic structure in the aurora, Space Sc. Rev., 92, 423-533, 2000.

Tomczyk, S., McIntosh, S. W., Keil, S. L., Judge, P. G., Schad, T., Seeley, D. H., and Edmondson, J.: Alfvén Waves in the Solar Corona, Science, 317, 1192-1196, 2007.

Treumann, R. A.: Theory of super-diffusion for the magnetopause, Geophys. Res. Lett., 24, 1727-1730, 1997.

Treumann, R. A.: Kinetic theoretical foundation of Lorentzian statistical mechanics, Phys. Scripta, 59, 19 , doi:10.1238/Physica.Regular.059a00019, 1999.

Tu, C.-Y., Marsch, E., and Thieme, K. M.: Basic properties of solar wind MHD turbulence near $0.3 \mathrm{AU}$ analyzed by means of Elsaesser variables, J. Geophys. Res., 94, 11739-11759, 1989.

Turner, N. J. and Sano, T.: Dead zone accretion flows in protostellar disks, Astrophys. J., 679, L131-L134, 2008.

Turner, N. J., Sano, T., and Dziourkevitch, N.: Turbulent mixing and the dead zone in protostellar disks, Astrophys. J., 659, 729-737, 2007.

Vasconcelos, M. J., Jatenco-Pereira, V., and Opher, R.: Alfvénic heating of protostellar accretion disks, Astrophys. J., 534, 967975, 2000.

Vasyliunas, V. M.: Low-energy electrons on the day side of the magnetosphere, J. Geophys. Res., 73, 7519-7523, 1968.

Verheest, F.: Nonlinear dust modes, Space Sci. Rev., 68, 109-114, 1994.

Verheest, F.: Waves in dusty space plasmas, Astrophys. Space Sc. Library, Kluwer Academic Publishers, Dordrecht, Vol. 245, 2000.

Verheest, F.: Nonlinear evolution equations for parallel electromagnetic multispecies plasma modes: Reductive perturbation theory in the linear wave frame and electric field generation, Phys. Scripta, T113, 92-96, 2004.

Verheest, F. and Cattaert, T.: Nonlinear electromagnetic modes in astrophysical plasmas with dust distributions, Astron. Astrophys., 421, 17-21, 2004.

Verheest, F. and Meuris, P.: Nonlinear electromagnetic modes in plasmas with variable dust charges, Phys. Lett. A, 210, 198-201, 1996.

Vidotto, A. A. and Jatenco-Pereira, V.: The effects of Alfvén waves and radiation pressure in dusty winds of Late-Type stars. II. Dustcyclotron damping, Astrophys. J., 639, 416-422, 2006.

Vladimirov, S. V.: Plasma Collective Effects in the Presence of Dust, Astrophys. Space Sc., 256, 85-105, 1997.

Voitenko, Y., Goossens, M., Sirenko, O., and Chian, A. C.-L.: Nonlinear excitation of kinetic Alfvén waves and whistler waves by electron beam-driven Langmuir waves in the solar corona, Astron. Astrophys., 409, 331-345, 2003.

Wahlund, J. E., Louarn, P., Chust, T., de Feraudy, H., Roux, A., Holback, B., Dovner, P. O., and Holmgren, G.: On ion acoustic turbulence and the nonlinear evolution of kinetic 
Alfvén waves in aurora, Geophys. Res. Lett., 21, 1831-1834, doi:10.1029/94GL01289, 1994.

Wahlund, J. E., Eriksson, A. I., Holback, B., Boehm, M. H., Bonnell, J., Kintner, P. M., Seyler, C. E., Clemmons, J. H., Eliasson, L., Knudsen, D. J., Norqvist, P., and Zanetti, L. J.: Broadband ELF plasma emission during auroral energization, 1, Slow ion acoustic waves, J. Geophys. Res., 103, 4343-4376, doi:10.1029/97JA02008, 1998.

Walk, R. M., Snyder, J. A., Scrivasan, P., Kirch, J., Diaz, S. O., Blanco, F. C., Shashurin, A., Keidar, M., and Sandler, A. D.: Cold atmospheric plasma for the ablative treatment of neuroblastoma, J. Pediatr. Surg., 48, 63-73, 2013.

Watt, C. E. J. and Rankin, R.: Electron acceleration due to inertial Alfvén waves in a non-Maxwellian plasma, J. Geophys. Res., 112, A04214, doi:10.1029/2006JA011907, 2007.

Wu, D. J. and Fang, C.: Tw-fluid motion of plasma in Alfvén waves and the heating of solar coronal loops, Astrophys. J., 511, 958964, 1999

Wu, D. J. and Fang, C.: Coronal plume heating and kinetic dissipation of kinetic Alfvén aves, Astrophys. J., 596, 656-652, 2003.

Wu, D. J. and Fang, C.: Sunspot chromospheric heating by kinetic Alfvén waves, Astrophys. J., 659, L181-L184, 2007.

Wu, D. J., Huang, G. L., Wang, D. Y., and Fälthammar, C. G.: Solitary kinetic Alfvén waves in the two-fluid model, Phys. Plasmas, 3, 2879-2884, 1996.
Wygant, J. R., Keiling, A., Cattell, C. A., Johnson, M., Lysak, R., Temerin, M., Mozer, F. S., Kletzing, C. A., Scudder, J. D., Peterson, W., Russell, C. T., Parks, G., Brittnacher, M., Germany, G., and Spann, J.: Polar spacecraft based comparisons of intense electric fields and Poynting flux near and within the plasma sheet-tail lobe boundary to UVI images: An energy source for the aurora, J. Geophys. Res., 105, 18675-18692, 2000.

Yang, X., Wang, C.-L., Liu, C.-B., Zhang, J.-R., Shi, Y.-R., Duan, W.-S., and Yang, L.: The collision effect between dust grains and ions to the dust ion acoustic waves in a dusty plasma, Phys. Plasmas, 19, 103705, doi:10.1063/1.4757637, 2012.

Yukhimuk, A., Fedun, V., Sirenko, O., and Voitenko, Yu.: Excitation of fast and slow magnetosonic waves by kinetic Alfvén waves, AIP Conf. Proc., 537, 311, doi:10.1063/1.1324955, 2000.

Zhao, J. S., Wu, D. J., and Lu, J. Y.: Kinetic Alfvén waves excited by oblique magnetohydrodynamic Alfvén waves in coronal holes, Astrophys. J., 735, 114, doi:10.1088/0004-637X/735/2/114, 2011.

Zouganelis, I.: Measuring suprathermal electron parameters in space plasmas: Implementation of the quasi-thermal noise spectroscopy with kappa distributions using in situ Ulysses/URAP radio measurements in the solar wind, J. Geophys. Res., 113, A08111, doi:10.1029/2007JA012979, 2008.

Zubia, K., Rubab, N., Shah, H. A., Salimullah, M., and Murtaza, G.: Kinetic Alfvén waves in a homogeneous dusty magnetoplasma with dust charge fluctuation effects, Phys. Plasmas, 14, 032105, doi:10.1063/1.2710457, 2007. 